11 Nitsche, M.A. et al. (2003) Facilitation of implicit motor learning by weak transcranial direct current stimulation of the primary motor cortex in the human. J. Cogn. Neurosci. 15, 619-626

12 Iyer, M.B. et al. (2005) Safety and cognitive effect of frontal DC brain polarization in healthy individuals. Neurology 64, 872-876

13 Kincses, T.Z. et al. (2004) Facilitation of probabilistic classification learning by transcranial direct current stimulation of the prefrontal cortex in the human. Neuropsychologia 42, 113-117

14 Bindman, L.J. et al. (1962) Long-lasting changes in the level of the electrical activity of the cerebral cortex produced bypolarizing currents. Nature 196, 584-585
15 Terzuolo, C.A. and Bullock, T.H. (1956) The measurement of imposed voltage gradient adequate to modulate neuronal firing. Proc. Natl. Acad. Sci. U. S. A. 42, 687-693

16 Purpura, D.P. and McMurtry, J.G. (1965) Intacellular activities and evoked potential changes during polarization of motor cortex. J. Neurophysiol. 28, 166-185

1364-6613/\$ - see front matter (c) 2005 Elsevier Ltd. All rights reserved. doi:10.1016/j.tics.2005.09.001

Letters

\title{
Detecting cheaters
}

\section{Leda Cosmides and John Tooby}

Center for Evolutionary Psychology, University of California, Santa Barbara, CA 93016, USA

\section{Laurence Fiddick}

School of Psychology, James Cook University, Australia

\section{Gregory A. Bryant}

Center for Culture, Brain, and Development, Department of Psychology, University of California, Los Angeles, CA, USA

Successful scientific theories are those that have survived repeated attempts to falsify them, make detailed and novel predictions about previously unobserved phenomena that are subsequently confirmed, predict new observations that falsify rival theories, fit coherently and even deductively with other established theories, and economically account for large bodies of known phenomena. A number of evolutionary psychological hypotheses have met all the criteria of successful scientific theories, including our proposal that the human mind contains (1) an evolved neurocognitive system that is functionally specialized for reasoning about social exchange, with a subroutine for detecting cheaters (social contract theory (SCT) [1-8]), and (2) an evolved neurocognitive system that is specialized for reasoning about precautions in hazardous situations (hazard management theory (HMT) [8]). By contrast, the Buller-Fodor counterhypothesis, constructed post hoc to retrodict a small subset of our results, has been repeatedly and decisively falsified by many researchers over the past fifteen years. These diverse experimental falsifications (which Buller fails to disclose [9]) are not hidden in obscure sources, but are prominently featured in publications that Buller cites on other points. Specifically:

Because social contract rules involve the deontic concept of obligation, all social contracts are deontic rules (but, crucially, not all deontic rules are social contracts). Following Fodor, Buller claims (incorrectly) that the main source of evidence for a cheater-detection specialization involves experiments that compare deontic conditionals with indicative ones. He argues that deontic conditionals 'have' a different logical form from indicatives and when social contracts elicit better violation detection than indicative conditionals, this is a 'logic effect', general to deontic rules,

Corresponding author: Cosmides, L. (cosmides@psych.ucsb.edu)

Available online 29 September 2005 rather than a content effect specific to social contract rules. The Buller-Fodor hypothesis makes a clear prediction: good performance (high levels of violation detection) will be found across a broad range of deontic rules, rather than just among the narrower subsets of deontic rules, like social contracts and precautions, that were evolutionarily significant. This key prediction is demonstrably false. As numerous experiments show, subjects perform poorly on deontic rules - even natural, familiar ones - when these do not resemble social contracts or precautions (Ref. [2] pp. 229-233, 238-240, 243$253 ;[3,10]$.

Indeed, using SCT and HMT we were able to predict and produce patterns of reasoning strikingly at variance with any that had been observed before, as well as show that their appearance and disappearance parametrically tracks manipulations of several theoretical variables that are central to SCT and HMT but irrelevant to rival theories (including Buller's). For example, as predicted by SCT, identical social contracts elicit good performance when violations might be intentional (and so reveal cheaters), but elicit poor performance when violations are innocent mistakes (and therefore cannot) [4,7]. Because two identical social contracts must necessarily have the same deontic logical form, Buller's hypothesis (incorrectly) predicts uniform and high performance across both conditions.

Similarly, social exchanges by their nature restrict access to benefits, and so one can transform an otherwise identical deontic rule from a social contract into an ordinary permission rule by parametrically changing the permitted action from a benefit to something neutral or unpleasant. As predicted by SCT (but contrary to Buller's hypothesis), performance plummets on the deontic rule to the degree the permitted action becomes less interpretable as a benefit [3]. 
If a single deontic reasoning system handles deontic social contracts and deontic precautions, then performance on both should be impaired by neural damage to that system. But if they are handled by two distinct neurocognitive systems, as predicted by SCT and HMT, then neural trauma could cause a dissociation. Buller's hypothesis fails again: focal brain damage can selectively impair social contract reasoning while leaving precautionary reasoning intact [5]. This dissociation within the domain of deontic rules has recently been replicated using neuroimaging [6].

Interpretations of social contract rules track SCT's domain-specialized inference procedures: in [8], we refuted Buller-style logic explanations of social contract results for perspective change, switched rules, and 'wants' problems - facts he fails to mention, let alone discuss. Buller's systematic inattention to large bodies of findings that conflict with his assertions is not due to lack of space in TICS - the pretence that these findings do not exist pervades his book, and its treatment of many areas of evolutionary psychology. (For further analysis, see www. psych.ucsb.edu/research/cep/buller.htm)

\section{References}

1 Cosmides, L. and Tooby, J. (2005) Neurocognitive adaptations designed for social exchange. In Evolutionary Psychology Handbook (Buss, D.M., ed.), Wiley
2 Cosmides, L. (1989) The logic of social exchange: has natural selection shaped how humans reason? Studies with the Wason selection task. Cognition 31, 187-276

3 Cosmides, L. and Tooby, J. (1992) Cognitive adaptations for social exchange. In The Adapted Mind: Evolutionary Psychology and the Generation of Culture (Barkow, J., Cosmides, L. and Tooby, J., eds), Oxford University Press

4 Cosmides, L. and Tooby, J. (2000) The cognitive neuroscience of social reasoning. In The New Cognitive Neurosciences (2nd Edn) (Gazzaniga, M.S., ed.), pp. 1259-1270, MIT Press

5 Stone, V. et al. (2002) Selective impairment of reasoning about social exchange in a patient with bilateral limbic system damage. Proc. Natl. Acad. Sci. U. S. A. 99, 11531-11536

6 Fiddick, L., Spampinato, M.V. and Grafman, J. Social contracts and precautions activate different neurological systems: an fMRI investigation of deontic reasoning. NeuroImage (in press)

7 Fiddick, L. (2004) Domains of deontic reasoning: resolving the discrepancy between the cognitive and moral reasoning literatures. Q. J. Exp. Psychol. 57A, 447-474

8 Fiddick, L., Cosmides, L. and Tooby, J. (2000) No interpretation without representation: the role of domain-specific representations and inferences in the Wason selection task. Cognition 77, 1-79

9 Buller, D.J. (2005) Evolutionary psychology: the emperor's new paradigm. Trends Cogn. Sci. 9, 277-283

10 Manktelow, K. and Over, D. (1990) Deontic thought and the selection task. In Lines of Thought: Reflections of the Psychology of Thinking (Gilhooly, K. et al., eds), pp. 153-164, Wiley

1364-6613/\$ - see front matter (c) 2005 Elsevier Ltd. All rights reserved. doi:10.1016/j.tics.2005.09.005

\title{
The evolution of jealousy
}

\author{
David M. Buss ${ }^{1}$ and Martie Haselton ${ }^{2}$ \\ ${ }^{1}$ Department of Psychology, University of Texas, Austin, TX, USA \\ ${ }^{2}$ Communication Studies and Department of Psychology, University of California, Los Angeles, USA
}

Two decades ago, mainstream psychologists explained jealousy as a pathology, social construction, or by-product of capitalist society, manifested identically in men and women [1]. Evolutionary psychologists, in contrast, hypothesized that jealousy is an evolved adaptation, activated by threats to a valuable relationship, functioning to protect it from partial or total loss [2-4]. Because the reproductive consequences of infidelity and partner loss are parallel for men and women in some respects, and asymmetric in others, the sexes were predicted to have similar psychologies in some respects, and different psychologies where their adaptive problems recurrently diverged. This program initially focused on a few core design features of jealousy, but has since expanded to study many more.

Sexual similarities include the following. Jealousy (i) is an emotion designed to alert an individual to threats to a valued relationship, (ii) is activated by the presence of interested and more desirable intrasexual rivals, and (iii) functions, in part, as a motivational mechanism with behavioral output designed to deter 'the dual specters of

Corresponding author: Buss, D.M. (dbuss@psy.utexas.edu).

Available online 30 September 2005 infidelity and abandonment' ([1], p. 35). (iv) '[B] oth sexes are hypothesized to become distressed over sexual and emotional infidelity' because both forms of infidelity provide important cues to the loss of reproductively valuable resources ([4], p. 251). When there is a discrepancy in mate value, (v) the lower-value partner will experience more intense jealousy [1].

There are at least 13 distinct hypothesized sexdifferentiated design features, and 13 out of 13 have been confirmed empirically. Men and women differ psychologically in the weighting given to sexual and emotional cues that trigger jealousy, such that (i) men more than women become upset at signals of sexual infidelity, which portend both paternity uncertainty and loss of reproductive resources to a rival; and (ii) women more than men become upset at signals of a partner's emotional infidelity, which threaten a loss of commitment and resources to a rival $[1,5]$.

When jealousy is activated by interlopers, (iii) women become especially distressed by threats from physically attractive rivals, whereas (iv) men become especially distressed by rivals with more resources [6]. Within 